Ann. Génét. Sél. anim., I97I, 3 (2), I45-I5I.

\title{
GÉNÉTIQUE DES POPULATIONS DE CHATS DOMESTIQUES \\ DANS LA VALLÉE DE CHAMONIX (HAUTE-SAVOIE)
}

\author{
Ph. DREUX \\ Laboratoive de Zoologie, \\ École Normale Supérieure \\ 46, rue dUlm, Paris $\left(5^{\mathrm{e}}\right)$
}

RÉSUMÉ

Dans la vallée de Chamonix, 249 Chats examinés se sont révélés appartenir à une population panmictique. Les fréquences des gènes de coloration du pelage ont été estimées. Elles se révèlent en bon accord avec celles des populations déjà étudiées en Europe occidentale. Une brève comparaison est faite avec ces populations.

\section{INTRODUCTION}

Sur une suggestion de J. B. S. HALDANE, la première estimation de la fréquence des gènes dans une population de Chats domestiques a été faite par SEARLE à I ondres (I949). On a constaté par la suite que les résultats de tels sondages pouvaient constituer un matériel de choix en génétique des populations, en particulier pour l'étude de la sélection. Aussi, les travaux analogues se sont multipliés de façon coordonnée, pour avoir de tels résultats dans toutes les régions du globe. Beaucoup sont encore toutefois à peu près inexplorées à ce point de vue, et la plus grande densité de sondages à l'heure actuelle se situe aux U.S.A. (ToDD, I969) et en Europe occidentale où ont été étudiées successivement un certain nombre de populations : à Paris (DREUX, I967 a), dans un district rural de Mayenne (DrEuX, I968 a), à Venise (SEARlE in DREuX, I967 b), en Angleterre (Robinson et Silson, I969). D'autres populations sont encore en cours d'étude. Le présent article expose les résultats obtenus à Chamonix, station touristique bien connue, et dans ses environs. 


\section{MÉTHODES}

L'étude génétique d'une population de Chats dans son ensemble est évidemment presque impossible à réaliser. Il faut donc faire un échantillonnage le plus nombreux possible en examinant un nombre important d'individus dont on note les différentes caractéristiques phénotypiques, qui donnent des renseignements sur leur génotype. Un tel examen est très commode à faire dans une fourrière ou une clinique vétérinaire. Toutefois, dans ce dernier cas, il se présente de difficiles problèmes d'échantillonnage. Il est en effet très possible que les Chats d'une clientèle vétérinaire aient pu être l'objet par leurs possesseurs d'une sélection, fondée sur la couleur, certains chatons pouvant être gardés comme animaux familiers de préférence à d'autres esthétiquement moins plaisants. De plus, beaucoup sont castrés et ne peuvent plus alors intervenir dans la reproduction. Une analyse fine des sous-populations d'adultes et de jeunes peut cependant permettre de tourner plus ou moins ces difficultés.

Lorsqu'il n'existe pas d'institution où l'on peut examiner un grand nombre de Chats, morts ou vivants, il est nécessaire de procéder à un sondage direct, ce qui a été le cas à Chamonix. L'observateur s'attache alors à visiter le plus grand nombre possible d'endroits et à repérer individuellement tous les Chats qu'il rencontre; un tel travail a été fait à Chamonix et dans ses environs, au cours de 5 séjours d'été consécutifs, de 1966 à 1970 (Dreux, 1968b, 1969a, 1969b).

Dans un sens, cette méthode est bien meilleure que la précédente, car elle étudie directement la population des animaux qui évoluent plus ou moins librement et prennent une part active à la reproduction, ce qui est le but souhaité. En revanche elle exige un observateur très exercé à l'approche des Chats et à la reconnaissance de leurs phénotypes. Beaucoup d'animaux sont assez farouches, ne peuvent être approchés de près, ce qui empêche souvent de déterminer leur sexe. De plus, il importe de ne pas compter plusieurs fois le même individu, ou de ne pas en confondre plusieurs qui se ressemblent. Heureusement, c'est plus facile qu'on ne pourrait le penser : les Chats ont en effet un polymorphisme si prononcé qu'il n'est pas trop difficile au cours d'un séjour de quelques semaines d'en connaître un grand nombre individuellement. De plus, ils sont casaniers et ne sortent pas d'un territoire assez restreint qui peut être facilement repéré.

D’une année sur l'autre, il est nécessaire de noter avec soin les Chats vus précédemment pour ne pas les confondre avec ceux qui sont nouvellement apparus. Une étude portant sur 5 étés a montré (chose assez surprenante pour une espèce dont la durée de vie peut atteindre une dizaine d'années) que la population se renouvelait chaque année dans une proportion qui pouvait atteindre $80 \mathrm{p}$. 100 . La mortalité des Chats adultes semble donc très élevée.

Toutes les précautions ont été prises pour éviter de compter plusieurs fois le même animal, que ce soit la même année ou deux années différentes. S'il y avait un doute, même léger, on comptait toujours un seul individu. La question ne s'est d'ailleurs pas posée dans beaucoup de hameaux ou d'endroits éloignés du centre de Chamonix qui n'ont été visités qu'une fois au cours de cette investigation.

Les Chats examinés dans ces lieux plus éloignés représentent environ 25-p. 100 du total des individus; ils ne diffèrent pas, de manière significative, de ceux de la zone centrale, semi-urbanisée, mais comprenant une grande proportion d'espaces verts. Au bout de cinq ans, il n'est probablement pas exagéré de dire que tous les Chats notés individuellement formaient une fraction très importante de l'ensemble de la population, certainement plus de la moitié, ce qui constitue un échantillonnage rarement réalisé.

\section{RÉSULTATS}

249 Chats en tout ont été examinés. 74 ont été reconnus comme nuâles, 82 comme femelles, ce qui donne pour le sex-ratio une valeur ne différant pas significativement de 0,$5 ; 93$ n'ont pu être approchés d'assez près pour pouvoir déterminer leur sexe. Aucun mâle castré n'a été reconnu de façon certaine. Sur ces 249 Chats, 72 étaient des chatons ou des jeunes n'ayant pas atteint l'âge adulte; leur nombre est trop faible pour pouvoir faire des comparaisons statistiquement valables avec les adultes; ils leur ont donc été réunis.

Le polymorphisme de ces Chats a été examiné par rapport aux facteurs génétiques suivants (RobINSON, I959; SEARLE, I968; Committee on nomenclature, rg68) :

- $W$, blanc dominant, 
- $O$, orange, partiellement dominant, lié au sexe, épistatique: sur $a$,

- $a$, non agouti, épistatique sur $t$,

- $t^{b}$, tigré large, allèle de $t^{+}$, tigré ordinaire,

$-d$, dilution maltaise,

- ch, chinchilla,

- $S$, panachure blanche irrégulière, semi-dominant.

L'allèle $T^{a}$ du locus $t$ (Abyssinie) n'a pas été rencontré. Un seul Chat siamois $c^{\mathrm{s}} c^{\mathrm{s}}$, d'ailleurs panaché de blanc, donc bâtard, a été rencontré; visiblement issu d'un croisement très consanguin, on n'en a pas tenu compte pour ne pas donner une valeur manifestement trop élevée du gène $c^{s}$. Aucune anomalie squelettique (syndactyle, polydactyle, queue tordue) n'a été remarquée.

Sur ces 249 individus, on a observé 7 [ $W$ ], blancs dominants; 242 pouvaient donc être classés par rapport au gène orange 0.207 étaient normalement colorés, I8 présentaient le phénotype orange et $\mathrm{I} 7$ le phénotype écaille-de-tortue $[0 /+]$. Comme le sexe d'une forte proportion d'individus n'était pas connu, le plus sûr est d'utiliser la méthode de probabilité maximum. Si 1'on suppose la population panmictique avec un sex-ratio de 0,5 , en appelant $q$ la fréquence du gène $O$, $p$ celle du gène + aver $p+q=\mathrm{r}$, les fréquences des 3 sortes de phénotypes seront :

— pour [O] : moyenne de $q\left(\delta^{3}\right)$ et de $q^{2}$ (कo) soit $\frac{q(\mathrm{I}-q)}{2}$,

- pour $[O /+]: \frac{2 p q}{2}=q(\mathrm{I}-q)$ (कq seules),

- pour [+] : moyenne de $p\left(\sigma^{*}\right)$ et de $p^{2}$ (qo) soit :

$$
\frac{\mathrm{I}-q+(\mathrm{I}-q)^{2}}{2}=\frac{2-3 q+q^{2}}{2}=\frac{(2-q)(\mathrm{I}-q)}{2}
$$

Le calcul montre que le maximum de vraisemblance pour l'obtention de ces classes de phénotypes est obtenu pour $q=0$,IO2 que l'on prendra comme fréquence $d u$ gène orange. Pour tester la panmixie de la population par rapport à ce gène, on a calculé les nombres théoriques des 3 phénotypes, indiqués entre parenthèses dans le tableau I à la suite du nombre observé. Le $\chi^{2}$ est égal à 2,9 pour I degré de liberté et l'bypothèse de la panmixie est tout à fait vraisemblable, bien qu'il y ait un excès de phénotypes orange et un déficit d'écaille-de-tortue. Ceci est du reste fréquent chez les populations déjà étudiées et semble bien causé par la sélection humaine; c'est par exemple le cas à Londres (SEARLE, I949) et à Paris (DREUx, Ig67 a).

\section{TABLEAU I}

Distributions observées et calculées (entre parenthèses) des phénotypes correspondant au gène orange pour la population de Chamonix

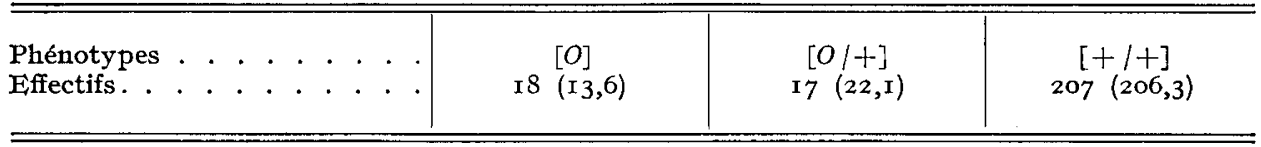


La population ayant été trouvée panmictique par rapport au gène $O$, on a supposé qu'elle l'était par rapport aux autres gènes de coloration, ce qui permet de calculer leurs fréquences en appliquant la loi de HARDY. Ces fréquences sont données dans le tableau 2. On y a ajouté celle de $O$ et celle de $l$ (poils longs); ce dernier gène semble bien en effet être récessif, quoique son expressivité soit assez variable.

TABLEAU 2

Fréquence des gènes dans la population de Chats de Chamonix

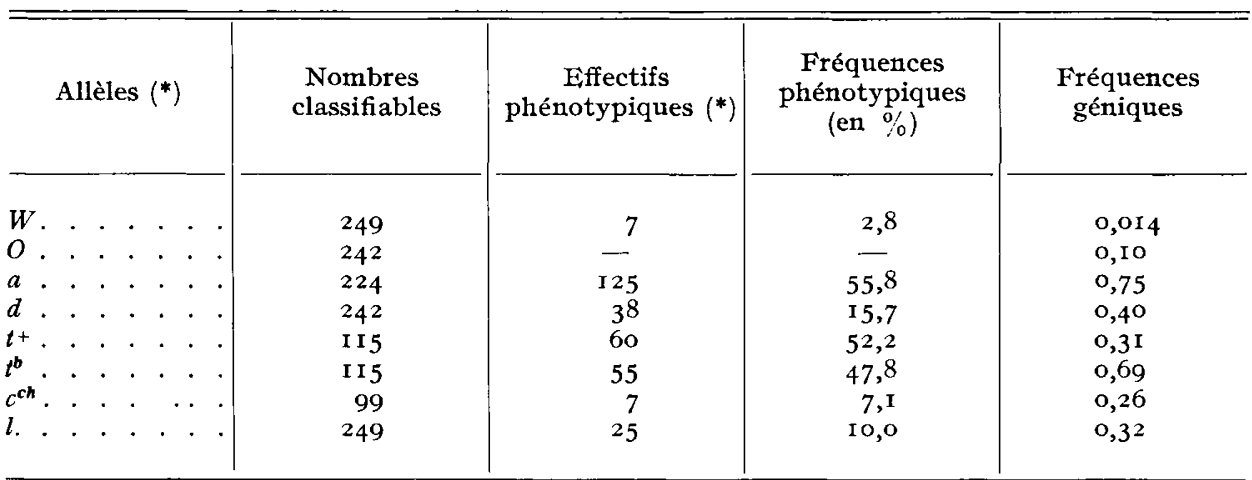

(*) Les effectifs phénotypiques observés sont ceux de l'homozygote pour les gènes récessifs (exemple: $a a)$, celui de l'hétérozygote ou de l'homozygote pour les gènes dominants (exemple $[W]$ ).

On a noté en plus sur les Chats non $[W]$ la panachure repérée suivant l'échelle définie précédemment (DREUX, I $967 a$ ) de o à 0,9 , les nombres exprimant à peu près la proportion de la surface blanche (tabl. 3). Il a paru utile aussi de noter les individus non-agouti (les seuls sur lesquels cela soit visible) et non porteurs de la panachure irrégulière qui possédaient sous la gorge et sous le ventre une petite tache de poils blancs bien visible, déjà nommée panachure $\varepsilon$. Elle était sûrement présente chez 57 Chats au moins sur un total de 78 . Le déterminisme de cette panachure discrète est encore inconnu alors qu'on sait que la panachure irrégulière est due à un gène $S$ semi-dominant.

TABLEAU 3

Degrés de panachure dans la population des Chats de Chamonix

\begin{tabular}{r|c|c|c|c|c|c|c|c|c|c}
\hline \hline Degrés de pana- & & & & & & & & & & \\
chure. . . . . & o & o, I & 0,2 & 0.3 & 0,4 & 0,5 & 0,6 & 0,7 & 0,8 & 0,9 \\
Effectifs...... & I 47 & 20 & I8 & 25 & 7 & 5 & 5 & 6 & 4 & 5 \\
\hline
\end{tabular}

Le tableau 3 permet d'évaluer la fréquence du gène $S$ dans la population; son allèle non panaché est homozygote chez I47 individus sur 242 , il a donc une fréquence de 0,78 et celle de $S$ est donc 0,22 . 


\section{DISCUSSION}

Il est intéressant de comparer ces fréquences géniques avec celles qui ont déjà été publiées en Europe occidentale, sans s'arrêter à celles d'autres régions du monde. Cette comparaison est présentée dans le tableau 4.

La première constatation qui s'impose est qu'en règle générale ces fréquences

TABIEAU 4

Fréquence des gènes dans les populations de Chats d'Europe occidentale

\begin{tabular}{|c|c|c|c|c|c|c|c|c|c|c|c|c|}
\hline & & & & & & & Chamonix & Paris & Londres & Venise & Mayenne & $\begin{array}{c}\text { Sud de } \\
\text { l'Angleterre }\end{array}$ \\
\hline$W$ & . & . & . & . & . & . & $0,0 I_{4}$ & $0,0 I_{1}$ & 0,004 & 0,003 & 0,006 & $0,0{ }_{4}$ \\
\hline$O$ & . & . & . & . & . & . & 0,10 & 0,06 & 0,1 I & 0,06 & 0,15 & 0,19 \\
\hline$a$. & . & . & . & . & . & . & 0,75 & $0,7 \mathrm{I}$ & 0,76 & 0,58 & 0,64 & 0,79 \\
\hline$d$ & . & . & . & . & . & . & 0,40 & 0,33 & $0, I_{4}$ & 0,35 & 0,29 & 0,26 \\
\hline$T^{a}$ & . & . & . & . & . & . & - & 0,001 & 0,002 & - & - & - \\
\hline$t^{+}$. & . & . & . & . & . & . & $0,3 \mathrm{I}$ & 0,22 & 0,19 & 0,52 & 0,39 & 0,16 \\
\hline$t^{b}$ & . & . & . & . & . & . & 0,69 & 0,78 & $0,8 \mathrm{I}$ & $0,4^{8}$ & $0,6 \mathrm{I}$ & 0,84 \\
\hline$c^{c h}$. & . & . & . & . & . & . & 0,26 & 0,24 & 0,20 & 0,60 & 0,23 & ? \\
\hline$c^{s}$. & . & . & . & . & . & . & - & 0,03 & - & 一 & - & - \\
\hline$S$. & . & . & . & . & . & . & 0,22 & 0,24 & 0,37 & 0,27 & 0,39 & 0,32 \\
\hline$l$. & . & . & . & . & . & . & 0,32 & 0,24 & 0,33 & $?$ & $0, I_{7}$ & 0,32 \\
\hline
\end{tabular}

sont très similaires. Malgré la distance qui sépare leurs lieux d'échantillonnage, encore trop peu nombreux malheureusement, les populations de Chats semblent bien appartenir à un même pool génique large et stable. C'est Venise qui s'écarte le plus de la moyenne par les basses fréquences de $a$ et $t^{\mathrm{b}}$ et la haute fréquence, surprenante, de $c^{\mathrm{ch}}$. Une anomalie très nette est la basse fréquence de la dilution maltaise, propre à Londres qui n'a pas d'ailleurs été confirmée par des sondages récents dans le Sud de 1'Angleterre (Robinson et Silson, I969).

On admet à l'heure actuelle que l'environnement urbain tend à défavoriser le gène $O$ et à favoriser $a$ et $t^{\mathrm{b}}$ (ToDD, I969) par des processus sélectifs encore discutés. C'est particulièrement net dans le cas de l'échantillonnage de Mayenne, réalisé en milieu rural, quand on le compare avec Londres et Paris, les villes étudiées les plus proches. Chamonix, bien que s'urbanisant progressivement, est encore assez proche de conditions rurales et, dans ces conditions, il est tout à fait naturel que les fréquences de $O$ et de $t^{\mathrm{b}}$ y soient intermédiaires entre celles de Paris et de la Mayenne. Mais, d'autre part, la fréquence de $a$ y est plus élevée même qu'à Paris, ce qui n'est guère en rapport avec l'hypothèse proposée.

Il faut bien remarquer qu'on ne possède guère de renseignements sur 1'origine et 1'évolution de cette population de Chamonix. Il est possible par exemple que beaucoup de Chats soient apportés des villes par des estivants, ce qui contribuerait à uniformiser les populations de France. 
Durant les 5 étés pendant lesquels les observations ont été poursuivies, les valeurs provisoires obtenues étaient notées au fur et à mesure (DREUX, I968 $b$, I969 $a$, r969 $b$ ). En général, elles n'ont fait qu'osciller au hasard des échantillonnages autour des valeurs définitives présentées dans le tableau 2 . Une seule exception s'est révélée, celle de la dilution maltaise $d$ dont la fréquence a augmenté d'une façon très régulière depuis $0,2 \mathrm{I}$ en I966 jusqu'à sa valeur définitive 0,40 . Il est évidemment difficile de savoir s'il s'agit d'une fluctuation passagère due au hasard et destinée à être suivie d'une fluctuation en sens inverse, ou bien s'il s'agit d'un véritable phénomène d'évolution de la population. Le gène $d$ serait dans ce dernier cas en train de subir une sélection positive dont il serait intéressant de connaître les causes. Des observations futures sont en tout cas nécessaires pour préciser la nature des phénomènes.

\section{CONCLUSION}

A mesure qu'on étudie davantage de populations de Chats dans 1'Europe occidentale, on se rend compte qu'elles restent assez similaires sur de grandes étendues, qu'elles sont stables et apparemment en équilibre. Il sera encore nécessaire d'accumuler de nombreux documents pour faire la part de la sélection humaine et pour la séparer de la sélection naturelle qui semble cependant jouer de façon indubitable. L'étude de ces mécanismes est encore sur de telles populations au stade du défrichement et on ne peut à l'heure actuelle que hasarder des hypothèses. L'action du milieu urbain sur les gènes $O, a$ et $t^{b}$ en est une, de même que celle de l'apparition assez récente de l'allèle $t^{\mathrm{b}}$, par une mutation qui semblerait bien être originaire d'Europe occidentale. C'est en effet dans ces régions que cet allèle présente les fréquences les plus elevées.

Reçu pour publication en mars 197 I.

\section{SUMMARY}

POPULATION GENETICS OF DOMESTIC CATS IN THE VALLEY OF CHAMONIX

(HAUTE-SAVOIE)

249 cats examined in the valley of Chamonix were found to belong to a panmictic population. Fur color gene frequency was estimated, and agrees well with that of western European populations already studied. These populations are briefly compared.

\section{RÉFÉRENCES BIBLIOGRAPHIQUES}

Committee on Standardized Genetic Nomenclature for Cats, 1968. Standardized genetic nomenclature for the domestic cat. J. Hered., 59, 39-40.

Dreux Ph., 1967a. Gene frequencies in the cat population of Paris. J. Hered., 58, 89-92.

DREuX Ph., I I967b. Génétique des populations de chats domestiques en Europe occidentale. Ann. Génét., 10, 141-145. 
DREUx Ph., 1968a. Gene frequencies in the cat population of a french rural district. J. Hered., 59, 37-39.

DREuX Ph., 1968b. Fréquence des gènes chez les chats de Chamonix (I). Carniv. Genet. Newsl,. (4), $62-63$.

DreuX Ph., I96ga. Fréquence des gènes chez les chats de Chamonix (II). Carniv. Genet. Nerwsl., (6), I2I.

DreuX Ph., 1969b, Fréquence des gènes chez les chats de Chamonix (III). Carniv. Genet. Newsl., (8), I76-I 78 .

Robinson R,. 1959. Genetics of the domestic cat. Biblio. Genet., 8, 273-362.

Robinson R., Silson M., I969. Mutant gene frequencies in cats of southern England. Theor. Appl. Genet., 39, 326-329.

SeARLE A. G., 1949. Gene frequencies in London's cats. J. Genet., 49, 214-220.

SeArLe A. G., 1968. Comparative genetics of coat colour in Mammals. Logos Press/Academic Press, 308 p., New York et London.

ToDd N. B., 1969. Cat gene frequencies in Chicago and other populations of the United States. J. Hered., 60, $273-277$. 